ARTICLE

https://doi.org/10.1038/s41467-019-12204-7

\title{
Switchable organoplatinum metallacycles with high quantum yields and tunable fluorescence wavelengths
}

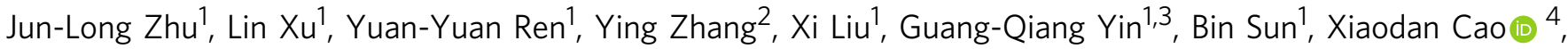
Zhuang Chen ${ }^{4}$, Xiao-Li Zhao ${ }^{1}$, Hongwei Tan², Jinquan Chen (1) ${ }^{4}$, Xiaopeng Li (i) ${ }^{3}$ \& Hai-Bo Yang ${ }^{1}$

The preparation of fluorescent discrete supramolecular coordination complexes (SCCs) has attracted considerable attention within the fields of supramolecular chemistry, materials science, and biological sciences. However, many challenges remain. For instance, fluorescence quenching often occurs due to the heavy-atom effect arising from the Pt(II)-based building block in Pt-based SCCs. Moreover, relatively few methods exist for tuning of the emission wavelength of discrete SCCs. Thus, it is still challenging to construct discrete SCCs with high fluorescence quantum yields and tunable fluorescence wavelengths. Here we report nine organoplatinum fluorescent metallacycles that exhibit high fluorescence quantum yields and tunable fluorescence wavelengths through simple regulation of their photoinduced electron transfer (PET) and intramolecular charge transfer (ICT) properties. Moreover, 3D fluorescent films and fluorescent inks for inkjet printing were fabricated using these metallacycles. This work provides a strategy to solve the fluorescence quenching problem arising from the heavy-atom effect of $\mathrm{Pt}(\mathrm{II})$, and offers an alternative approach to tune the emission wavelengths of discrete SCCs in the same solvent.

\footnotetext{
${ }^{1}$ Shanghai Key Laboratory of Green Chemistry and Chemical Processes, School of Chemistry and Molecular Engineering, East China Normal University, 3663N Zhongshan Road, Shanghai 200062, China. ${ }^{2}$ College of Chemistry, Beijing Normal University, Beijing 100050, China. ${ }^{3}$ Department of Chemistry, University of South Florida, Tampa, FL 33620, USA. ${ }^{4}$ State Key Laboratory of Precision Spectroscopy, East China Normal University, Shanghai 200062, China. Correspondence and requests for materials should be addressed to L.X. (email: Ixu@chem.ecnu.edu.cn) or to H.-B.Y. (email: hbyang@chem.ecnu.edu.cn)
} 
O ver the past three decades, coordination-driven selfassembly, which is based on metal-ligand coordination interaction, has evolved to be a well-established methodology for constructing supramolecular coordination complexes (SCCs) such as one-dimensional (1-D) helices, two-dimensional (2-D) polygons, and even three-dimensional (3-D) polyhedra ${ }^{1-10}$. A variety of elegant and sophisticated SCCs have been fabricated through coordination-driven self-assembly, which have displayed extensive applications in host-guest chemistry ${ }^{11-13}$, sensing ${ }^{14-16}$, catalysis ${ }^{17-21}$, smart polymeric materials ${ }^{22,23}$, and biomedicines $^{24-26}$. Recently, the preparation of fluorescent discrete SCCs has garnered great attention owing to their promising applications such as chemical sensors, optical devices, supramolecular biomedicines, and so on ${ }^{27-37}$. Moreover, the presence of chromophores in SCCs allows for real-time monitoring the selfassembly process and dynamics of the resultant SCCs by highly sensitive fluorescence technique ${ }^{38}$. Generally, two main methodologies have been explored for constructing fluorescent SCCs. One methodology is the direct introduction of chromophore as the core skeleton of the discrete $\mathrm{SCCs}^{39,40}$. For instance, Mukherjee et al. reported the construction of a hexagonal fluorescent SCC through the self-assembly of porphyrin-based tetratopic donor (5,10,15,20-tetrakis(4-pyridyl)porphyrin) and $90^{\circ} \mathrm{Pt}$ (II)-based acceptor, which displayed high encapsulating efficiency toward $\mathrm{Zn}^{2+39}$. The other methodology is the preparation of discrete fluorescent SCCs by encapsulating fluorescent dyes inside the cavity of $\mathrm{SCCs}^{41,42}$. For example, Yoshizawa and coworkers constructed a series of fluorescent SCCs upon encapsulation of various fluorescent dyes such as BODIPY or coumarin derivatives by using a supramolecular coordination capsule ${ }^{41}$.

Although fruitful achievements have been gained in the development of fluorescent discrete SCCs, many problems are still far from being fully resolved in this area. For example, the heavy-atom effect arising from the $\mathrm{Pt}(\mathrm{II})$-based building block sometimes induces a significant increase in the amounts of intersystem crossing (ISC) owing to spin-orbit coupling, which thus leads to an obvious fluorescence quenching of the discrete SCCs ${ }^{43,44}$. Therefore, compared with their precursor building blocks, the fluorescence quantum yields of the discrete organoplatinum SCCs are relatively lower, which is usually unfavorable for their practical application. Second, there is a relative lack of efficient and simple strategy to tune the emission wavelength of the discrete SCCs ${ }^{27,45}$. It should be noted that the fluorescent materials with tunable emission wavelength have versatile applications as photovoltaics, light-emitting diodes, nonlinear optical materials, chemical sensors, and biological labels ${ }^{46-48}$. Up to now, changing the solvents is the most used method to tune the emission wavelength of the discrete SCCs since the emission wavelength of many fluorophores is often sensitive to the solvents. However, some solvents such as acetonitrile could disrupt the $\mathrm{Pt}-\mathrm{N}$ coordination bond because these solvents feature the stronger binding ability to platinum atom than pyridine ${ }^{38}$. Thus, in some cases, such strategy of changing the solvents exhibits certain limitation in tuning the emission wavelength of the discrete SCCs. Therefore, it is still challenging to construct discrete organoplatinum SCCs with high fluorescence quantum yields and tunable fluorescence emission wavelengths.

As two kinds of classic recognition and sensing mechanisms, photoinduced electron transfer (PET) and intramolecular charge transfer (ICT) have been widely exploited for detection of cations, anions, and small-molecules ${ }^{49,50}$. A typical PET molecule often includes three parts: a fluorophore that acts as the electron acceptor, a receptor that serves as an electron donor or a quencher, and a spacer that links the two parts of fluorophore and receptor (Supplementary Fig. 1-2). Fluorescent molecules based on PET are often structured as fluorophore-spacer-receptor constructs. In the PET system, the photoinduced electron transfer from the receptor to the fluorophore will induce fluorescence quenching. However, this PET process is restricted when the receptor binds upon its electron-withdrawing targets (such as metal ions), which thus induces the enhancement of fluorescence emission. However, fluorescent molecules on the basis of ICT are featured by conjugation of an electron-donating unit to an electron-accepting unit in one molecule to rise a "push-pull" $\pi$ electron system in the excited state (Supplementary Fig. 3). When the electron-accepting part interacts with an electronwithdrawing guest (such as metal ions), the electron-accepting character of the fluorescent molecule increases, thus generating a red shift in the emission spectrum. In contrast, an evident blue shift can be observed when the ICT becomes less developed owing to the interaction of the electron-donating part with an electron-withdrawing guest. Although PET and ICT mechanisms have been widely used to develop diverse sensors, probes, and molecular machine ${ }^{51-54}$, systematic investigation and development of fluorescent SCCs by using PET and ICT has not been reported yet. By taking the inherent advantages of PET and ICT, we envision that the construction of self-assembled organoplatinum metallacycles with high fluorescence quantum yields and tunable fluorescence wavelengths could be realized by means of reasonable PET and ICT strategies.

Herein, nine triarylamine-based dipyridyl building blocks with electron-withdrawing or electron-donating groups para to the tertiary amine core (L1-L9) were reasonably designed and synthesized (Fig. 1). Interestingly, the PET and ICT properties of these nine triarylamine-based dipyridyl building blocks could be switched based on the push-pull electronic effect of the substituents. Therefore, through coordination-driven self-assembly of these nine triarylamine-based dipyridyl building blocks with
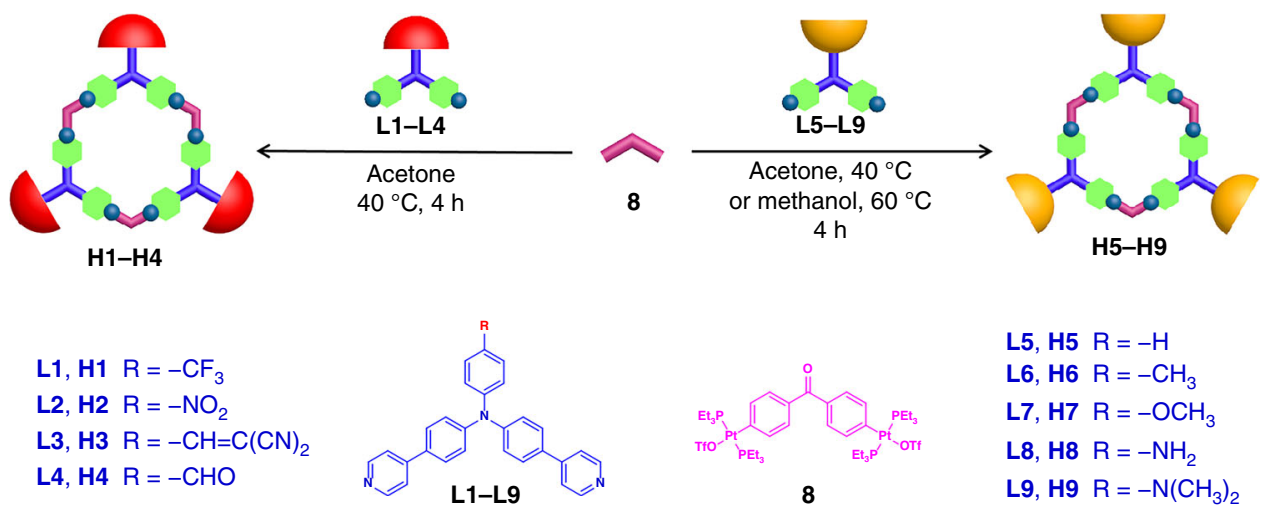

$$
\begin{aligned}
& \text { L5, H5 R }=-\mathrm{H} \\
& \text { L6, H6 R }=-\mathrm{CH}_{3} \\
& \text { L7, H7 R } \mathrm{R}=-\mathrm{OCH}_{3} \\
& \text { L8, H8 R }=-\mathrm{NH}_{2} \\
& \text { L9, H9 R } \mathrm{R}=-\mathrm{N}\left(\mathrm{CH}_{3}\right)_{2}
\end{aligned}
$$

Fig. 1 Self-assembly of $120^{\circ}$ triarylamine-based dipyridyl donor ligands L1-L9 and 120 di-Pt(II) acceptor 8 into hexagonal metallacycles H1-H9 
di-Pt(II) acceptor, nine fluorescent metallacycles with tunable fluorescence wavelengths (from $480 \mathrm{~nm}$ to $590 \mathrm{~nm}$ ) in the same solvent and high fluorescence quantum yields (up to 61\%) were easily prepared. In this study, we presented the example on the preparation of organoplatinum metallacycles with high quantum yields and tunable fluorescence wavelengths by simply switching the PET and ICT properties of building blocks based on the substituent effect. This PET/ICT switchable strategy not only offers a good guidance on how to avoid the heavy-atom effect arising from the platinum ion in the construction of fluorescent platinum(II)-pyridyl SCCs, but also provides an alternative approach to prepare fluorescent platinum(II)-pyridyl SCCs with high quantum yields and even tunable wavelength of the discrete SCCs without changing the solvents.

\section{Results}

Synthesis and characterization. With the aim of preparing a series of fluorescent metallacycles with the tunable fluorescence properties, nine $120^{\circ}$ triarylamine-based dipyridyl donor ligands L1-L9 with different pendant functional groups para to the tertiary amine core were designed and synthesized (Fig. 1 and Supplementary Fig. 189-197). Different pendant functional groups, such as $-\mathrm{CF}_{3},-\mathrm{NO}_{2},-\mathrm{CH}=\mathrm{C}(\mathrm{CN})_{2},-\mathrm{CHO},-\mathrm{H}$, $-\mathrm{CH}_{3},-\mathrm{OCH}_{3},-\mathrm{NH}_{2}$, and $-\mathrm{N}\left(\mathrm{CH}_{3}\right)_{2}$ were selected to control the push-pull electronic effect of the substituents, thereby switching the PET and ICT properties of the building blocks. All ligands L1-L9 were well characterized by using multinuclear NMR $\left\{{ }^{1} \mathrm{H}\right.$ and $\left.{ }^{13} \mathrm{C}\right\}$ spectroscopy and HR-MS (EI) spectrometry (Supplementary Fig. 4-23 and 84-93). Moreover, the structures of ligands L3, L4, L6, and L8 were unambiguously confirmed by means of single-crystal X-ray diffraction, which revealed that the angle between the two pyridyl rings was close to $120^{\circ}$ (Supplementary Fig. 185 and Supplementary Tables 1-4).

According to the design principles of coordination-driven selfassembly, the combination of three $120^{\circ}$ donor ligands and three $120^{\circ}$ acceptor ligands can result in the formation of a discrete hexagonal metallacycle. Stirring the mixtures of the $120^{\circ}$ triarylamine-based dipyridyl donor ligands L1-L7, and $\mathbf{L 9}$ with the $120^{\circ} \mathrm{di}-\mathrm{Pt}(\mathrm{II})$ acceptor 8 in a $1: 1$ ratio in acetone at $40^{\circ} \mathrm{C}$ for $4 \mathrm{~h}$ led to the formation of discrete hexagonal metallacycles H1H7, and H9, respectively (Fig. 1 and Supplementary Fig. 198206). It should be noted that the preparation of $\mathbf{H 8}$ was conducted through the reaction of ligands $\mathbf{L 8}$ and $\mathbf{8}$ in methanol at $60^{\circ} \mathrm{C}$ for $4 \mathrm{~h}$.

Multinuclear NMR $\left({ }^{1} \mathrm{H},{ }^{13} \mathrm{C},{ }^{31} \mathrm{P}, 2-\mathrm{D}\right.$ DOSY, ${ }^{1} \mathrm{H}-{ }^{1} \mathrm{H}$ COSY, and NOESY) analysis of the assemblies H1-H9 revealed the formation of discrete metallacycles with highly symmetric hexagonal scaffold (Supplementary Fig. 24-77). For instance, in the ${ }^{1} \mathrm{H}$ NMR spectrum of each assembly, the $\alpha$-hydrogen and $\beta$ hydrogen nuclei of the pyridine rings exhibited downfield shifts because of the loss of electron density that occurred upon coordination of the pyridine- $\mathrm{N}$ atom with the $\mathrm{Pt}(\mathrm{II})$ metal center. As shown in the ${ }^{1} \mathrm{H}$ NMR spectrum of metallacycle $\mathbf{H 4}$, the $\alpha$ - and $\beta$-pyridyl hydrogen signals both exhibited the obvious downfield shifts (for $\alpha-\mathrm{H}, \Delta \delta \approx 0.04 \mathrm{ppm}$, for $\beta-\mathrm{H}, \Delta \delta \approx 0.45 \mathrm{ppm}$ ), which were attributed to the formation of platinum-nitrogen $(\mathrm{Pt}-\mathrm{N})$ bonds (Fig. 2a). All ${ }^{31} \mathrm{P}\left\{{ }^{1} \mathrm{H}\right\}$ NMR spectra of metallacycles H1H9 displayed a sharp singlet (for example, ca. 13.73 ppm for $\mathbf{H 4}$ shifted upfield from the precursor 8 by $\sim 6.05 \mathrm{ppm}$, Fig. $2 \mathrm{~b}$ ). This change, as well as the decrease in the coupling of the flanking ${ }^{195} \mathrm{Pt}$ satellites, was consistent with back-donation from the platinum atoms. The sharp NMR signals in both the ${ }^{31} \mathrm{P}$ and ${ }^{1} \mathrm{H}$ NMR spectra of metallacycles H1-H9 along with the good solubility ruled out the formation of oligomers for each assembly. Moreover, for all nine metallacycles H1-H9, only one set of signals was observed in 2-D DOSY spectra for each metallacycle, thus indicating the existence of the sole species. Further characterization of all metallacycles $\mathbf{H 1}-\mathbf{H} \mathbf{9}$ by ${ }^{1} \mathrm{H}-{ }^{1} \mathrm{H}$ COSY and NOESY exhibited obvious cross peaks between the signals of the pyridine protons $(\alpha-\mathrm{H}$ and $\beta-\mathrm{H})$ and the $\mathrm{PEt}_{3}$ protons $\left(-\mathrm{CH}_{2}\right.$ and $-\mathrm{CH}_{3}$ ), which showed good agreement with the formation of the discrete metallacycles $\mathbf{H 1 - H 9}$ based on the formation of $\mathrm{Pt}-\mathrm{N}$ bonds.

Investigation of electrospray ionization time-of-flight mass spectrometry (ESI-TOF-MS) provided further support for the formation of discrete hexagonal metallacycles H1-H9 (Supplementary Fig. 94-102). For instance, as shown in Fig. 2c, the mass spectrum of metallacycle $\mathbf{H 4}$ displayed two main peaks at $\mathrm{m} / z=$ 1177.06 and $m / z=911.91$, corresponding to different charge states resulted from the loss of trifluoromethanesulfonate counterions $\left[\mathrm{M}-4 \mathrm{OTf}^{-}\right]^{4+}$ and $\left[\mathrm{M}-5 \mathrm{OTf}^{-}\right]^{5+}$, respectively, where $\mathrm{M}$ represents the intact assembly. The isotopic resolution of each peak agreed well with the theoretical distribution, which allowed for the molecularity of the hexagonal metallacycle to be unambiguously established.

All attempts to grow X-ray-quality single crystals of these hexagonal metallacycles H1-H9 have so far proven unsuccessful. Therefore, the PM6 semiempirical molecular orbital method was utilized to acquire further insight into the structural characteristics of these hexagonal metallacycles. Molecular simulation indicated that all hexagonal metallacycles H1-H9 exhibited a very similar and roughly planar hexagonal ring at their core surrounded by three triarylamine units with an internal diameter of $~ 3.36 \mathrm{~nm}$ (Supplementary Fig. 158-162).

Photophysical investigations. The photophysical properties of metallacycles H1-H9 were then investigated. As shown in Fig. 3a and Supplementary Fig. 105-123, most of metallacycles exhibited two sharp absorption bands or a main band companied with a shoulder peak centered at $\sim 375 \mathrm{~nm}$. The high-energy absorption bands were almost unaffected by the nature of pendant functional groups para to the tertiary amine core. However, unlike the highenergy absorption bands, the low-energy absorption bands of metallacycles $\mathbf{H 1 - H 9}$ were very sensitive to the nature of pendant functional groups para to the tertiary amine core, which endows the maximal absorption wavelengths of metallacycles H1-H9 ranging from $390 \mathrm{~nm}$ to $450 \mathrm{~nm}$. Although the similar phenomenon was observed in the absorption spectra of ligands L1-L9, most of metallacycles H1-H9 displayed appreciable red-shifts in the absorption spectra when compared with their corresponding ligands L1-L9. For instance, the lowest-energy absorption band for ligand $\mathbf{L} 5$ was centered at $361 \mathrm{~nm}$, whereas the metallacycle H5 constructed from this ligand L5 had a band centered at 414 $\mathrm{nm}$. The observed results were attributed to the coordination of metal center with the pyridyl nitrogen, which enriched the ligand $\pi$-system, lowered the energy required for excitation, and helped to stabilize the $\mathrm{LUMO}^{43}$.

The fluorescence emission spectra of ligands L1-L9 as well as metallacycles H1-H9 were measured with the results being shown in Fig. 3 b and Supplementary Fig. 105-123. The emission spectra for both ligands L1-L9 and metallacycles H1-H9 exhibited single bands with the maximum emission wavelength of metallacycles H1-H9 ranging from $480 \mathrm{~nm}$ to $590 \mathrm{~nm}$. In addition, it was worth noting that the color of the fluorescence of all ligands was markedly changed upon the formation of metallacycles (Fig. 3c, f). For instance, the solution of ligand L1 emitted blue fluorescence while metallacycle $\mathbf{H} \mathbf{1}$ displayed green fluorescence in the same solvent. Interestingly, the metallacycles H1-H4 with electron-withdrawing groups para to the tertiary amine core displayed the higher emission intensity compared 

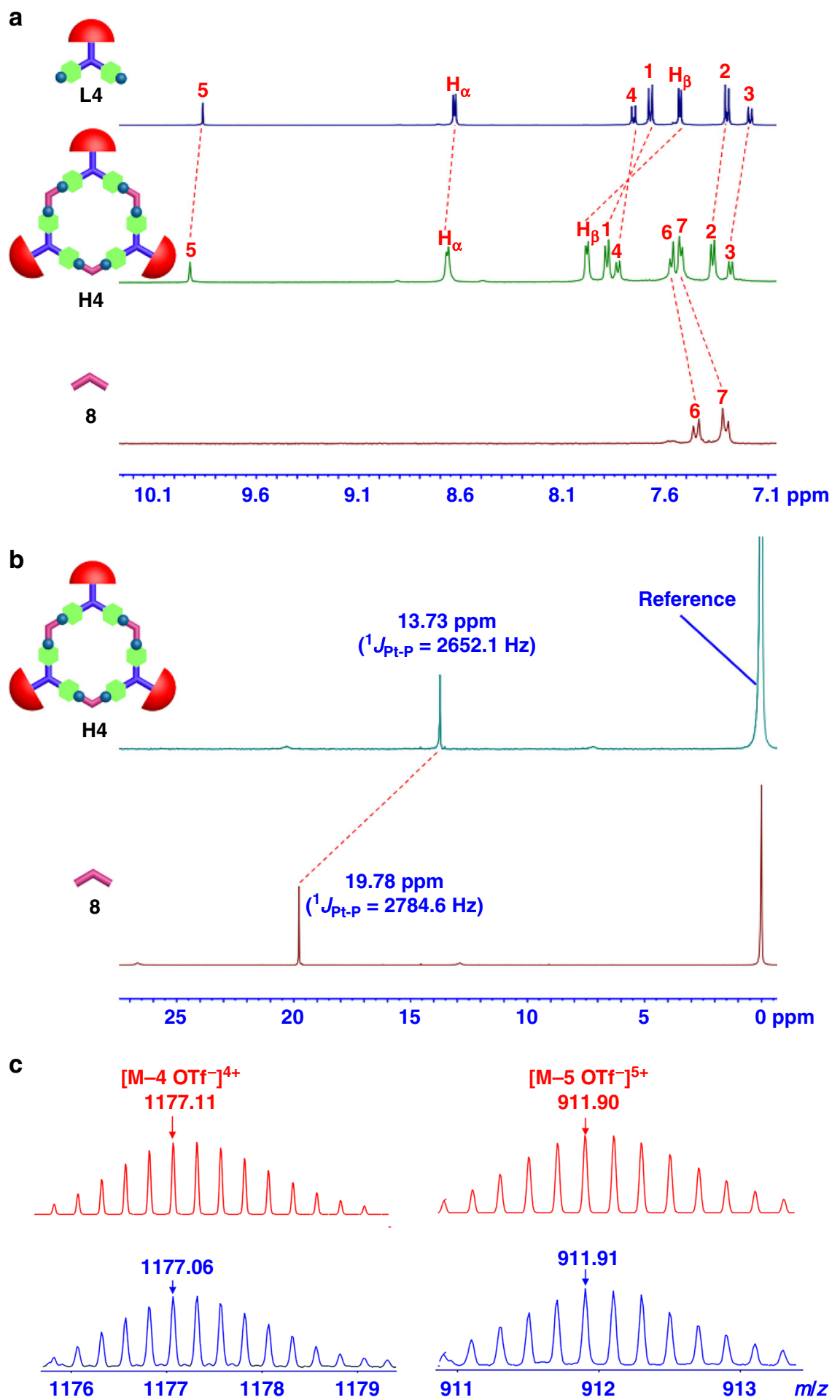

Fig. 2 NMR spectra and ESI-TOF-MS spectra of $\mathbf{L 4}$ and $\mathbf{~ H 4}$. a Partial ${ }^{1} \mathrm{H}$ NMR spectra $\left(500 \mathrm{MHz}\right.$, in $\mathrm{CD}_{2} \mathrm{Cl}_{2}$ ) of ligand $\mathbf{L 4}$ (top), $120^{\circ}$ dipyridine donor 8 (bottom), and metallacycle $\mathbf{H 4}$ (middle). b ${ }^{31} \mathrm{P} \mathrm{NMR}$ spectra $\left(202 \mathrm{MHz}\right.$, in $\mathrm{CD}_{2} \mathrm{Cl}_{2}$ ) of metallacycle $\mathbf{H 4}$ (top) and $120^{\circ}$ dipyridine donor 8 (bottom). c Theoretical (top) and experimental (bottom) ESI-TOF-MS spectra of metallacycle $\mathbf{H} 4$

with their constituent ligands L1-L4 (3.0 equivalents of ligand relative to each corresponding metallacycle). However, unlike H1-H4, the metallacycles H5-H9, which contained electrondonating groups para to the tertiary amine core, exhibited the lower emission intensity compared with their constituent ligands L5-L9. In order to obtain a deeper insight into the photophysical properties of ligands L1-L9 and metallacycles H1-H9, threedimensional excitation-emission matrix (3-D EEMs) technology was employed to investigate the emission spectra. As shown in Supplementary Fig. 186, the samples mappings were collected in all ligands L1-L9 and metallacycles H1-H9. It could be found that the formation of metallacycles resulted in an apparent change in the EEM peaks. For example, the formation of metallacycle $\mathbf{H} \mathbf{1}$ led to a red shift in the peak wavelengths $\left(E_{\mathrm{x}} / E_{\mathrm{m}}\right)$ from $341 / 420 \mathrm{~nm}$ to $400 / 518 \mathrm{~nm}$. However, as the formation of metallacycle $\mathbf{H} \mathbf{2}$, the fluorescence maximum was red-shifted from 

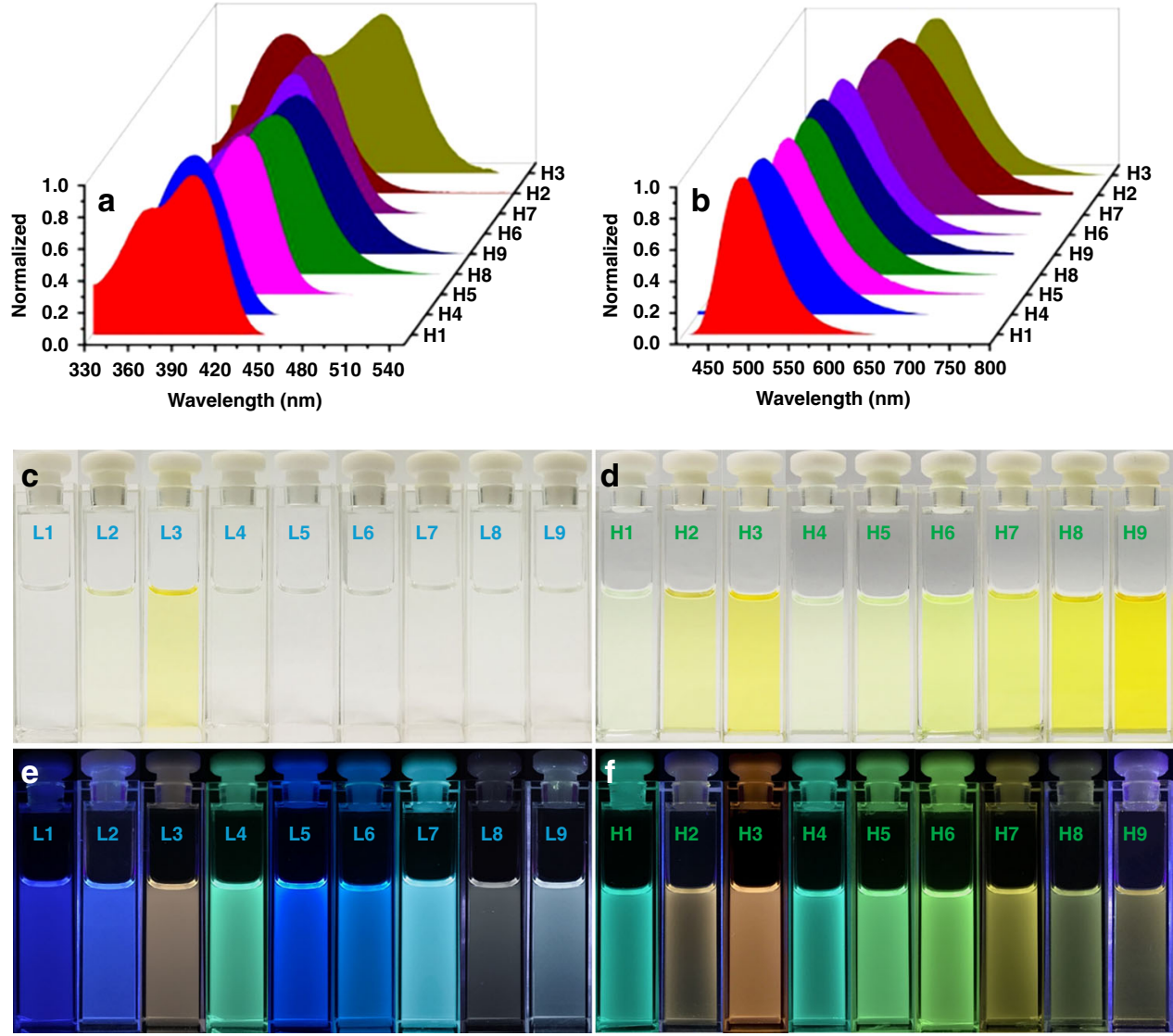

Fig. 3 Absorption, emission spectra, and photographs of L1-L9 and H1-H9. Normalized absorption $\mathbf{a}$ and fluorescence emission $\mathbf{b}$ spectra of metallacycles H1-H9 in dichloromethane. Photographs of ligands L1-L9 in dichloromethane in visible light $\mathbf{c}$ and under $365 \mathrm{~nm}$ light excitation e. Photographs of metallacycles H1-H9 in dichloromethane in visible light $\mathbf{d}$ and under $365 \mathrm{~nm}$ light excitation $\mathbf{f}$

\begin{tabular}{|c|c|c|c|c|}
\hline Substituent & Ligand & Fluorescence quantum yield (\%) & Metallacycle & Fluorescence quantum yield (\%) \\
\hline$-\mathrm{CF}_{3}$ & $\mathbf{L 1}$ & 56 & H1 & 61 \\
\hline$-\mathrm{CH}=\mathrm{C}(\mathrm{CN})_{2}$ & $\mathbf{L 3}$ & 13 & H3 & 18 \\
\hline$-\mathrm{CHO}$ & L4 & 41 & H4 & 53 \\
\hline$-\mathrm{H}$ & $\mathbf{L 5}$ & 57 & H5 & 55 \\
\hline$-\mathrm{NH}_{2}$ & $\mathbf{L 8}$ & 2.5 & H8 & 2.0 \\
\hline$-\mathrm{N}\left(\mathrm{CH}_{3}\right)_{2}$ & L9 & 1.1 & H9 & 0.5 \\
\hline
\end{tabular}

$E_{\mathrm{x}} / E_{\mathrm{m}} 354 / 455 \mathrm{~nm}$ to $E_{\mathrm{x}} / E_{\mathrm{m}} 412 / 572 \mathrm{~nm}$ with the difference in $E_{\mathrm{x}} /$ $E_{\mathrm{m}}$ wavelengths as much as $58 / 117 \mathrm{~nm}$. Different $E_{\mathrm{x}} / E_{\mathrm{m}}$ wavelength shifts produced by the formation of different metallacycle suggested that the tuning of the emission wavelengths could be achieved. Moreover, 3-D EEMs measurements of ligands L1-L9 and metallacycles H1-H9 were consistent with the $1-\mathrm{D}$ fluorescence results and no other light-emitting species was observed, which demonstrated that the metallacycles H1-H9 showed the independence of emission from excitation.

As mentioned above, generally, the fluorescence quantum yields of platinum(II)-pyridyl SCCs are lower than their precursor ligands mainly owing to the well-known heavy-atom effect of platinum. The fluorescence quantum yields of the ligands L1-L9 and metallacycles H1-H9 were determined as shown in Table 1. Unlike the previously reported platinum(II)-pyridyl SCCs whose fluorescence quantum yields were usually lower than $20 \%$ even if the fluorescence quantum yields of their constituent ligands exceed 50\%, metallacycles H1, H4, H5, and H6 showed high fluorescence quantum yields of $61 \%, 53 \%, 55 \%$, and $52 \%$, respectively. More importantly, as expected, upon the formation of hexagonal metallacycles, the enhancement of fluorescence quantum yields was observed for metallacycles $\mathbf{H 1}, \mathbf{H 3}$, and $\mathbf{H 4}$ containing electron-withdrawing groups para to the tertiary amine core because the PET process from pyridine to fluorophore was blocked by coordination of the pyridines to the platinum atoms. Moreover, in these PET systems, as pyridyls make little contribution to the fluorescence emission, the heavy-atom effect arising from the platinum ion could be avoided even under the formation of platinum(II)-pyridyl SCCs. Although metallacycle H2 contains electron-withdrawing group (nitro group) para to the tertiary amine core, metallacycle $\mathbf{H} \mathbf{2}$ displayed an extreme low fluorescence quantum yield $(\sim 1 \%)$ similar to that of its 


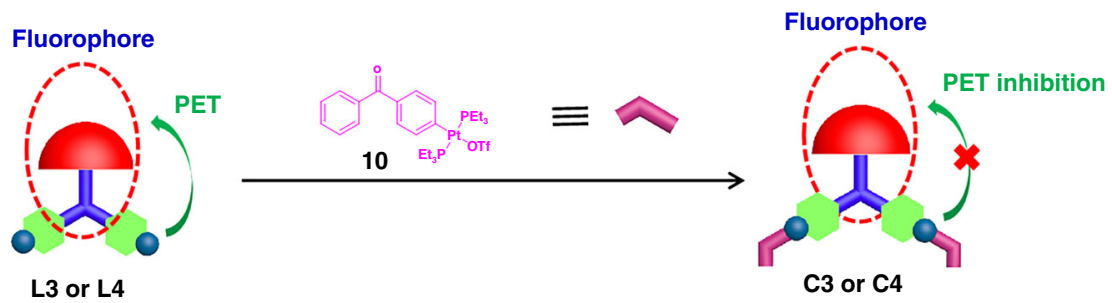

Fluorescence enhancement

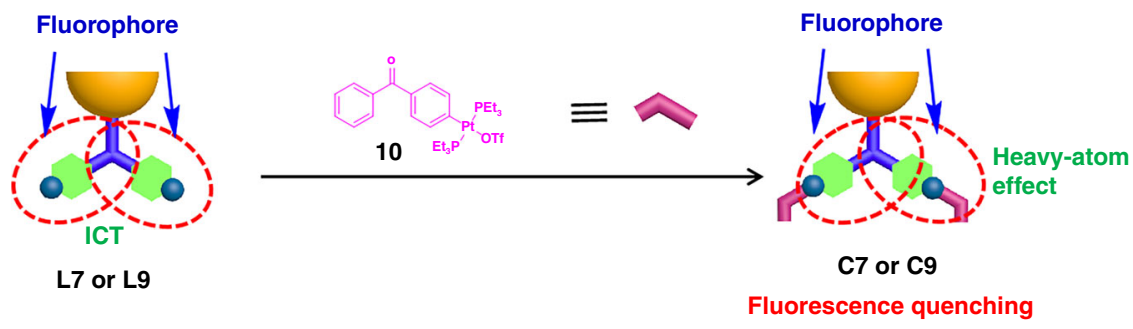

Fig. 4 Cartoon illustration of the mechanisms of fluorescence changes of ligands $\mathbf{L 3}, \mathbf{L 4}$, $\mathbf{2 7}$, and $\mathbf{L} \mathbf{9}$ responding to mono- $\mathrm{Pt}(\mathrm{II})$ ligand $\mathbf{1 0}$

constituent ligand L2 ( 2\%). This observation was attributed to the presence of nitro-groups in the triarylamine skeleton of $\mathbf{H} \mathbf{2}$ and $\mathbf{L} 2$ that caused the usual decrease of radiative deactivation in the singlet state in favor of significant ISC to the triplet state, thus quenching the fluorescence. The similar nitro group effect has been previously reported in literatures ${ }^{55}$. For the metallacycles H5-H9 containing electron-donating groups, para to the tertiary amine core, the fluorescence quantum yields of them were determined to be lower than those of their free ligands, which was consistent with a Pt-enhanced ISC rate as well as the enhanced ICT owing to the presence of the heavy metal ions. Therefore, these results suggested that the emission wavelengths of metallacycles H1-H9 were largely depended upon their substituents, which induced the change of emission colors of metallacycles $\mathbf{H 1}-\mathbf{H} 9$ from blue to green to orange by simply varying the functional groups para to the tertiary amine core. Moreover, the fluorescence quantum yields of metallacycles H1H9 could be also finely tuned up to $61 \%$. More importantly, by modulating the electron-withdrawing groups or electrondonating groups, the decrease or even increase in the fluorescence quantum yields of metallacycles $\mathbf{H 1 - H 9}$ could be regulated as well when compared with their constituent ligands.

Mechanism studies. In order to ensure that such changes in the emission wavelengths and intensities were caused by the platinum (II)-pyridyl coordination, the control experiment was conducted by adding mono-Pt(II) ligand $\mathbf{1 0}$ into the solution of ligands L1L9, and meanwhile, the monitoring of the changes in both absorption and fluorescence spectra were accomplished. As mono-Pt(II) ligand 10 only contains one platinum, it cannot selfassemble with ligands L1-L9 to form a series of hexagonal metallacycles but can coordinate with them to generate $[2+1]$ model complexes (Fig. 4). Ligands L3, L4, L7, and L9 were selected as the representative ligands since two of them contain electron-withdrawing groups para to the tertiary amine core and the other two ligands contain electron-donating groups para to the tertiary amine core. The fast formation of $[2+1]$ model complexes $\mathbf{C} 3, \mathbf{C} 4, \mathbf{C} 7$, and $\mathbf{C} \mathbf{9}$ through the reaction of ligands $\mathbf{L} 3$, L4, L7, and $\mathbf{L 9}$ with mono-Pt(II) ligand 10 was confirmed by multinuclear NMR $\left({ }^{1} \mathrm{H}\right.$ and $\left.{ }^{31} \mathrm{P}\right)$ titration experiments (Supplementary Fig. 125-132) and ESI-TOF-MS spectra (Supplementary Fig. 133-136). Thus, the absorption and fluorescence responses of ligands L3, L4, L7, and $\mathbf{L 9}$ to mono-Pt(II) ligand 10 were investigated. As shown in Fig. 5, the gradual addition of 2.0 equivalents of mono-Pt(II) ligand $\mathbf{1 0}$ into the solution of ligand L3 or L4 induced the marked increase of fluorescence intensity. The fluorescence enhancement for the formation of $[2+1]$ model complex can be explained by the inhibition of PET process from pyridines to luminescent moiety. However, an obvious red shift in the absorption wavelength and a decrease in the emission intensity were observed when 2.0 equivalents of mono-Pt(II) ligand $\mathbf{1 0}$ was gradually added into the solution of ligand L7 or L9, respectively. The red shift of wavelengths was attributed to the enhancement of push-pull electronic properties of luminescent molecule. More importantly, the changes in the emission wavelengths and intensities were consistent with those of the formation of metallacycles, which suggested that the changes in photophysical properties were achieved through the coordination of platinum(II) and pyridyl. Furthermore, the absorption and fluorescence responses of ligands $\mathbf{L 3}, \mathbf{L 4}, \mathrm{L} 7$, and $\mathrm{L} 9$ to mono-Pt (II) ligand $\mathbf{1 0}$ were in accord with classical PET and ICT mechanisms, respectively, which gave further support for our proposed mechanisms.

With the aim to gaining more insight into the mechanisms for the changing of the fluorescence quantum yields of metallacycles H1-H9 upon the formation of metallacycles, time-resolved intensity decays of both ligands L1-L9 and metallacycles H1H9 were measured by time-correlated single-photon counting (TCSPC) method (Supplementary Fig. 137-138 and Supplementary Tables 7-8). The fluorescence lifetime measurements of ligands L1-L9 and metallacycles $\mathbf{H 1 - H 9}$ revealed that the fluorescence intensity decays were multiexponential and all of them were within nanosecond scale, thus suggesting a typical fluorescent emission. For instance, the decay of metallacycle H1 was found to follow a biexponential function with a shorter component of $1.6 \mathrm{~ns}$ with a $35 \%$ proportion and a longer component of $2.4 \mathrm{~ns}$ with a $65 \%$ proportion. However, the decay of metallacycle $\mathbf{H} \mathbf{3}$ was found to follow a triexponential function with the components of $0.1 \mathrm{~ns}, 1.4 \mathrm{~ns}$, and $2.4 \mathrm{~ns}$ with the proportions of $22 \%, 26 \%$, and $52 \%$, respectively. To avoid the possible complexity, an averaged fluorescence lifetime $(\tau)$ of each of the ligands L1-L9 and metallacycles H1-H9 was considered rather than emphasizing too much on the individual components. From the average fluorescence lifetimes $(\tau)$ of ligands L1-L9 and metallacycles H1-H9 shown in Supplementary Tables 7-8, the radiative rate constant $k_{\mathrm{rad}}$ and the nonradiative rate constant $k_{\mathrm{nr}}$ were calculated based on following photophysical equations:

$$
k_{\mathrm{exc}}=1 / \tau
$$



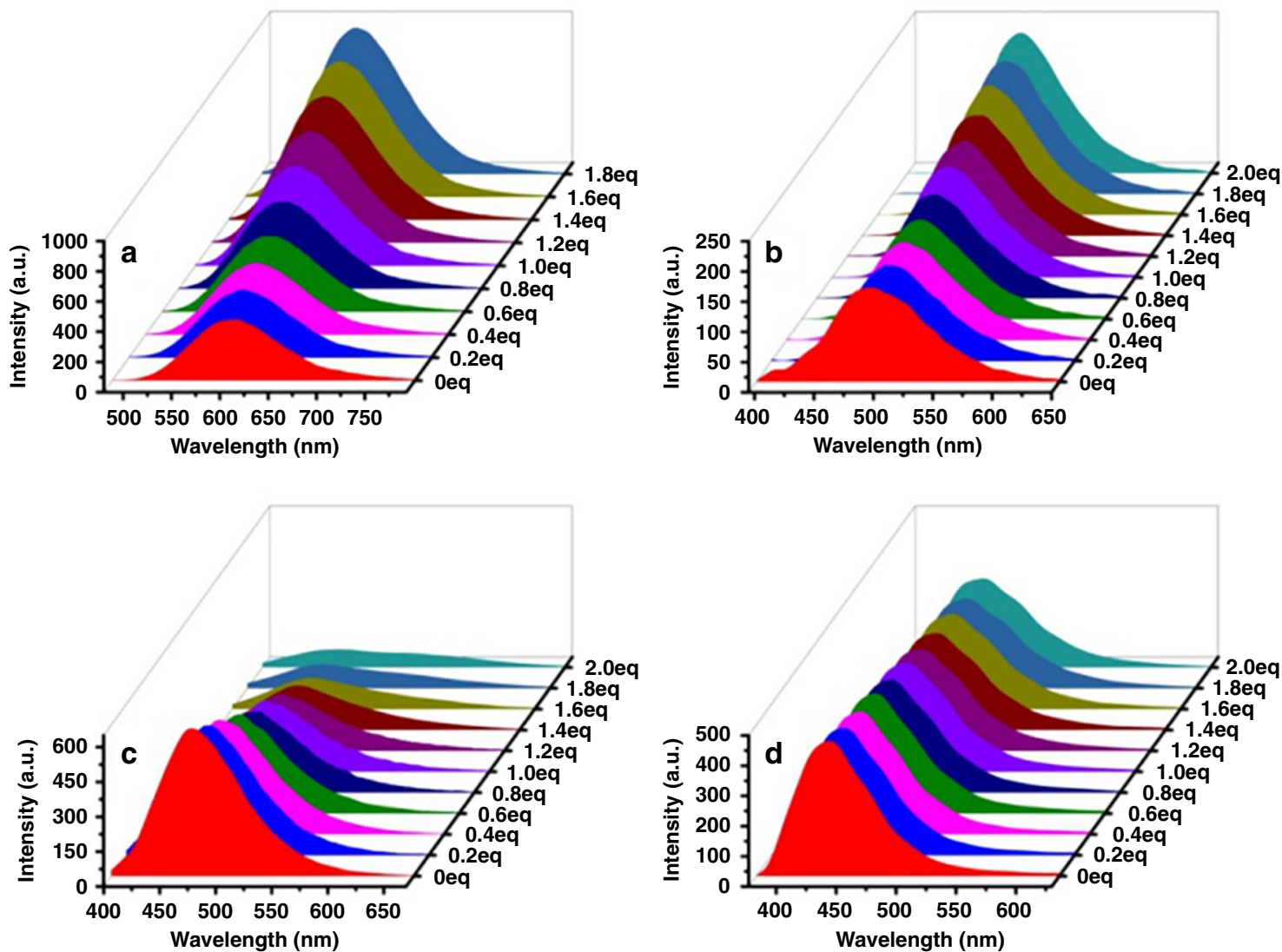

Fig. 5 Fluorescence titration spectra of ligands $\mathbf{L 3}, \mathbf{L 4}, \mathbf{L 7}$, and $\mathbf{L 9}$. Fluorescence spectra of ligands $\mathbf{L 3} \mathbf{a}, \mathbf{L} \mathbf{4} \mathbf{b}, \mathbf{L 7} \mathbf{c}$, and $\mathbf{L 9} \mathbf{d}$ upon the addition of mono-Pt (II) ligand $\mathbf{1 0}$ in dichloromethane

Table 2 Excitation rate constants $\left(k_{\text {exc }}\right)$, radiative rate constants $\left(k_{\text {rad }}\right)$, and nonradiative rate constants $\left(k_{\text {nr }}\right)$ for ligands L1-L9 and metallacycles $\mathrm{H} 1-\mathrm{H} 9$

\begin{tabular}{|c|c|c|c|c|c|c|c|c|}
\hline Substituent & Ligand & $k_{\text {exc }}\left(s^{-1}\right)$ & $k_{\mathrm{rad}}\left(\mathrm{s}^{-1}\right)$ & $k_{\mathrm{nr}}\left(\mathrm{s}^{-1}\right)$ & Metallacycle & $k_{\text {exc }}\left(s^{-1}\right)$ & $k_{\text {rad }}\left(s^{-1}\right)$ & $k_{\mathrm{nr}}\left(\mathrm{s}^{-1}\right)$ \\
\hline$-\mathrm{CF}_{3}$ & $\mathbf{L 1}$ & $6.25 \times 10^{8}$ & $3.48 \times 10^{8}$ & $2.77 \times 10^{8}$ & H1 & $4.55 \times 10^{8}$ & $2.77 \times 10^{8}$ & $1.77 \times 10^{8}$ \\
\hline$-\mathrm{NO}_{2}$ & L2 & $4.00 \times 10^{8}$ & $8.56 \times 10^{6}$ & $3.91 \times 10^{8}$ & H2 & $1.67 \times 10^{9}$ & $1.00 \times 10^{7}$ & $1.66 \times 10^{9}$ \\
\hline$-\mathrm{CH}=\mathrm{C}(\mathrm{CN})_{2}$ & L3 & $5.26 \times 10^{8}$ & $6.83 \times 10^{7}$ & $4.58 \times 10^{8}$ & H3 & $4.76 \times 10^{8}$ & $8.63 \times 10^{7}$ & $3.90 \times 10^{8}$ \\
\hline$-\mathrm{H}$ & $\mathbf{L 5}$ & $4.55 \times 10^{8}$ & $2.61 \times 10^{8}$ & $1.94 \times 10^{8}$ & H5 & $3.85 \times 10^{8}$ & $2.10 \times 10^{8}$ & $1.75 \times 10^{8}$ \\
\hline$-\mathrm{CH}_{3}$ & $\mathbf{L 6}$ & $3.85 \times 10^{8}$ & $2.30 \times 10^{8}$ & $1.55 \times 10^{8}$ & H6 & $3.23 \times 10^{8}$ & $1.69 \times 10^{8}$ & $1.54 \times 10^{8}$ \\
\hline$-\mathrm{OCH}_{3}$ & $\mathbf{L 7}$ & $2.56 \times 10^{8}$ & $1.40 \times 10^{8}$ & $1.16 \times 10^{8}$ & H7 & $1.25 \times 10^{9}$ & $1.10 \times 10^{8}$ & $1.14 \times 10^{9}$ \\
\hline
\end{tabular}

$$
\begin{aligned}
& k_{\mathrm{rad}}=\Phi_{\mathrm{F}} * k_{\mathrm{exc}} \\
& k_{\mathrm{exc}}=k_{\mathrm{rad}}+k_{\mathrm{nr}}
\end{aligned}
$$

where $k_{\text {exc }}$ was excitation rate constant, $k_{\text {rad }}$ and $k_{\mathrm{nr}}$ represented the radiative rate constant and nonradiative rate constant, respectively. For each ligand or metallacycle, $k_{\mathrm{exc}}$ could be calculated from Eq. (1), and $k_{\text {rad }}$ could be measured from Eq. (2) using the experimentally determined fluorescence quantum yield $\left(\Phi_{\mathrm{F}}\right)$ value. Finally, once the $k_{\mathrm{exc}}$ and $k_{\mathrm{rad}}$ were known, the $k_{\mathrm{nr}}$ could be deduced from Eq. 3. As shown in Table 2, for example, although the nonradiative rate constants $\left(k_{\mathrm{nr}}\right)$ of metallacycles H5-H8 were not larger than those of their corresponding ligands, the value of the ratio nonradiative rate constant $\left(k_{\mathrm{nr}}\right)$ /radiative rate constant $\left(k_{\mathrm{rad}}\right)$ of each metallacycle (H5-H8) was larger than that of the corresponding ligand, which provided a quantitative understanding of the lower quantum yields of metallacycles H5H8 compared with their constituent ligands L5-L8. Therefore, the obtained $k_{\text {rad }}$ and $k_{\text {nr }}$ afforded quantitative insights into the reasons for the changing of the fluorescence quantum yields of metallacycles $\mathbf{H 1}-\mathbf{H} 9$ upon the formation of metallacycles.

With the aim of confirming whether the emission properties of metallacycles $\mathbf{H 5}-\mathbf{H} 9$ is fluorescence or phosphorescence, the delayed emission spectra of representative metallacycles H5-H9 were measured. As shown in Supplementary Fig. 141, very weak emission from metallacycles $\mathbf{H 5}-\mathbf{H} 7$ and almost no emission from metallacycles H8-H9 were observed. Moreover, the normalized steady-state emission spectra of metallacycles H5H7 were coincident with those of their delayed emission spectra (Supplementary Fig. 142). Furthermore, the emission lifetime measurements of metallacycles $\mathbf{H 5}-\mathbf{H} \mathbf{9}$ revealed that all of them 

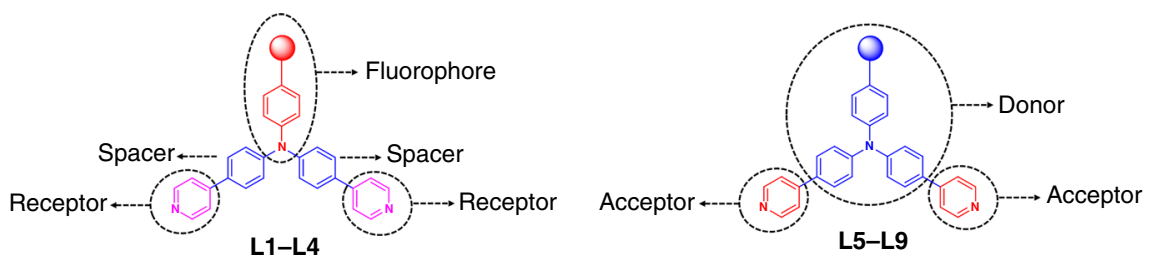

Fig. 6 Schematic illustrations of PET/ICT of ligands L1-L9

were within nanosecond scale. The results further indicated that the emission properties of metallacycles $\mathbf{H 5}-\mathbf{H} \mathbf{9}$ is fluorescence rather than phosphorescence.

In order to further elucidate the mechanism and the effect of substituted groups in the tuning of fluorescence emission wavelength and fluorescence quantum yield, molecular simulation by using time-dependent density functional theory (TDDFT) were carried out for both ligands L1-L9 and metallacycles H1-H9. As shown in Supplementary Fig. 163-180, for ligands L2-L4, which contained electron-withdrawing groups para to the tertiary amine core, their emission was mainly generated from the part of aniline and electron-withdrawing groups as witness by most of the electron densities of HOMO and LUMO were localized on the part of aniline and electron-withdrawing groups. Notably, ligand $\mathbf{L} \mathbf{1}$ and metallacycle $\mathbf{H} \mathbf{1}$ were exceptions, in which most of the electron densities were localized on the part of aniline and pyridine units although they were also substituted by electron-withdrawing group (trifluoromethyl), which might be caused by the well-known "fluorine effect". In these ligands L2$\mathbf{L 4}$, the part of aniline and electron-withdrawing groups acted as a fluorophore, pyridine units served as a receptor, and benzene units were the spacer that linked the two parts of fluorophore and receptor (Fig. 6). It indicated that they were PET molecules. Without binding of platinum(II), PET process occurred owing to the transfer of electron density, originating at the lone pair electrons on $\mathrm{N}$ atoms of the pyridine units, to the LUMO of the fluorophore. However, binding of platinum(II) to pyridine during the formation of metallacycles $\mathbf{H 3}-\mathbf{H} \mathbf{4}$ hindered the PET process, thus leading to the enhancement of fluorescence intensity and quantum yield. Although the molecular simulation results also demonstrated that the binding of platinum(II) to pyridine during the formation of metallacycle $\mathbf{H} \mathbf{2}$ hindered the PET process, both nitro group-containing metallacycle $\mathbf{H} 2$ and ligand $\mathbf{L} 2$ displayed extremely low quantum yields owing to the strong $\mathrm{S} 1 \rightarrow$ T1 ISC caused by nitro group. It should be noted that pyridine units in metallacycles $\mathbf{H 2}-\mathbf{H} \mathbf{4}$ just served as a receptor rather than a fluorophore in this case. Therefore, the heavy-atom effect arising from the platinum ion could be avoided even under the formation of platinum(II)-pyridyl SCCs.

For ligands L5-L9, which contained electron-donating groups para to the tertiary amine core, their emission was mainly generated from the part of aniline and pyridine units as the electron densities of HOMO of ligands L5-L9 were distributed over the whole molecule while most of the electron densities of LUMO were localized on the part of aniline and pyridine units. In these ligands L5-L9, the part of aniline and pyridine units acted as a fluorophore, in which aniline and pyridine units served as donor and acceptor, respectively (Fig. 6). It means that ligands L5-L9 were ICT molecules. Electron-donating groups such as $-\mathrm{H},-\mathrm{CH}_{3},-\mathrm{OCH}_{3},-\mathrm{NH}_{2}$, and $-\mathrm{N}\left(\mathrm{CH}_{3}\right)_{2}$ could regulate the electron-donating ability of aniline, and then changed the emission wavelength of these fluorescent ligands. When the electron-accepting part pyridine units interacted with platinum(II), the electron-accepting character of the fluorescent molecule increases, thus generating a red shift in the emission spectrum. In the ligands L5-L9, pyridine units were important part of fluorophore and made large contribution to the fluorescence emission. The coordination of platinum(II) with pyridine during the formation of metallacycles $\mathbf{H 5}-\mathbf{H} \mathbf{9}$ could induce the decrease of fluorescence intensity and fluorescence quantum yield due to the heavy-atom effect arising from the platinum ion.

In order to provide the more convincing molecular calculations, ligands L3 and L7 as well as metallacycles $\mathbf{H} \mathbf{3}$ and $\mathbf{H} 7$ were selected as the representatives to investigate the whole molecular simulation through the analysis of hole and electron distribution by using TD-DFT. As shown in Supplementary Fig. 181, for ligand L3, holes and electrons were mainly located at the part of aniline and electron-withdrawing groups, which indicated that electrons transferred from the aniline groups to the electronwithdrawing groups when ligand $\mathbf{L 3}$ was excited to the S1 state. However, for ligand L7, holes and electrons were distributed at the part of aniline and pyridine units (Supplementary Fig. 182). This result showed that electrons transferred from the aniline groups to the pyridine units when ligand L7 was excited to the S1 state. For metallacycle $\mathbf{H 3}$, the hole-electron distributions of S1, S2, S3 states, which derived from three ligands respectively, were similar with each other (Supplementary Fig. 183). More importantly, all of them were similar with the hole-electron distributions of S1 states of the corresponding ligand L3. These results suggested that electrons also transferred from the aniline groups to the pyridine units when excitation of metallacycle $\mathbf{H 3}$. Similar phenomena were also observed in metallacycle $\mathbf{H 7}$ (Supplementary Fig. 184).

More-detailed investigations of TD-DFT calculations were conducted by selecting ligand L3, metallacycle $\mathbf{H 3}$, ligand L7, and metallacycle $\mathbf{H 7}$ as representatives since they contain electronwithdrawing groups and electron-donating groups para to the tertiary amine core, respectively. The results were shown in Fig. 7 and Supplementary Table 11. In each case, the long wavelength absorption and emission were assigned to a transition between S0 $\leftrightarrow$ S1 (S0: ground-state, S1: singlet excited state), mainly corresponding to the promotion of an electron from HOMO to LUMO on the chromophore core. They were characteristic $\pi \rightarrow$ $\pi^{*}$ absorption and emission from the chromophore core. Moreover, the orbital transition from HOMO to $\mathrm{LUMO}+1$ was also observed in ligand L3, metallacycle $\mathbf{H 3}$, ligand L7, and metallacycle H7. For ligand L3 and metallacycle H3, although molecular orbital LUMO exhibited electron density centered on the part of aniline and electron-withdrawing groups, molecular orbital LUMO +1 displayed electron density mainly centered on the part of aniline and pyridine units. Supplementary Table 11 clearly illustrated that the oscillator strength $(f$, which is a very important parameter to evaluate the possibility of a transition) of the transition between HOMO and LUMO was bigger than that of the transition between HOMO and LUMO +1 in both ligand L3 and metallacycle H3, which indicated the emission of ligand L3 and metallacycle H3 was mainly generated from the part of aniline and electron-withdrawing groups and PET played more important role in tuning of the fluorescence emission wavelength and fluorescence quantum yield of ligand L3 and metallacycle H3. However, for ligand L7 and metallacycle H7, both molecular 
a

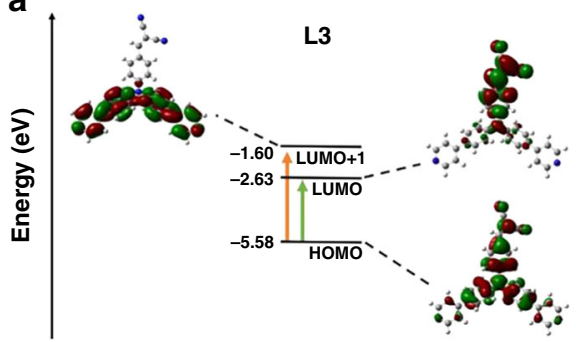

C

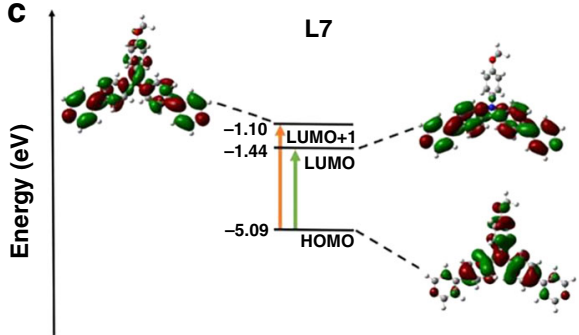

b

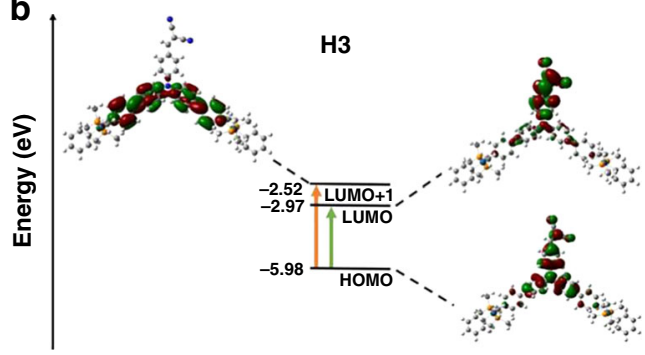

H7

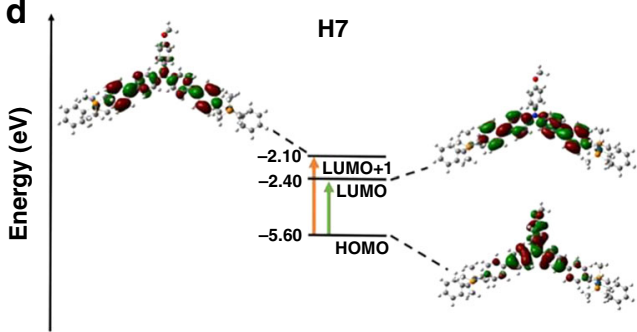

Fig. $\mathbf{7}$ TD-DFT transitions for $\mathbf{L 3}, \mathbf{L 7}, \mathbf{H 3}$, and $\mathbf{H 7}$. Predicted TD-DFT transitions for ligands $\mathbf{L 3} \mathbf{a}$, $\mathbf{L 7} \mathbf{c}$, and metallacycles $\mathbf{H 3} \mathbf{b}$, $\mathbf{H 7} \mathbf{d}$ model with oscillator strengths above 0.3

orbitals LUMO and LUMO + 1 were mainly localized on the part of aniline and pyridine units, which suggested that the ICT emission from aniline to pyridine units was the main factor in tuning of the fluorescence emission wavelength and fluorescence quantum yield of ligand L7 and metallacycle H7. These results further supported the mechanisms proposed above.

Moreover, in order to ensure that the enhancement of fluorescence for metallacycles $\mathbf{H 1}, \mathbf{H 3}$, and $\mathbf{H 4}$ were caused by the inhibition of PET process through the platinum(II)-pyridyl coordination, a representative control experiment was conducted by mixing dipyridyl ligand L3 and platinum-bromine complex 11 for $6.0 \mathrm{~h}$ (Supplementary Fig. 187). The platinum atoms in complex 11 were protected by bromine moieties, which cannot coordinate with ligand L3 to form a metallacycle. As shown in Supplementary Fig. 188, almost no change in the absorption and emission spectra of ligand L3 was observed, which further demonstrated that both reasons for inhibiting PET process and the driving force for metallacycle formation were the platinum (II)-pyridyl coordination.

The preparation of versatile fluorescent materials. By taking advantage of their excellent fluorescence properties, including tunable fluorescence wavelengths, tunable fluorescence quantum yields, and high emission, metallacycles $\mathbf{H 1 - H 9}$ were applied to prepare versatile fluorescent materials. The construction of dye-loaded thinlayer chromatography (TLC) plates has been attracted increasing interesting, as their strong mechanical strength, high portability, simple operability, and broad applications in the fluorescence sensing of explosive gases and volatile gases ${ }^{56,57}$. So, a series of fluorescent metallacycles H1-H9-loaded TLC plates were manufactured simply in the following way. Nine TLC plates were immersed into the dichloromethane solution of metallacycles $\mathbf{H 1 - H 9}$, respectively, and they were then dried in the air for several hours. As displayed in Fig. 8a, the prepared H1-H9-loaded plates emitted different color fluorescence upon excitation under a hand-held UV lamp with excitation wavelength at $365 \mathrm{~nm}$. For instance, the metallacycle H3-loaded plate emitted an orange fluorescence while the metallacycle $\mathbf{H 7}$-loaded plate emitted a green fluorescence.

Despite many organic materials exhibited high emission efficiency in solution, lots of them were non-emissive in the solid state because of the fluorescence quenching caused by intermolecular interactions occurring in the condensed phase ${ }^{58}$. Herein, metallacycles $\mathbf{H 1}, \mathbf{H} 3$, and $\mathbf{H} 7$ were selected as the representatives to investigate the fluorescence properties in the solid state through the preparation of fluorescent polymer films. It is known that the fluorescent polymer films feature some prominent advantages, such as reusability and easy fabrication into devices ${ }^{59,60}$. H1, H3, and $\mathbf{H 7}$-functionalized polymer films were obtained by dropping the DMF solution of metallacycles H1, H3, and H7 (0.1 wt $\%)$ doped with polyvinylidene fluoride (PVDF) onto the $\mathrm{SiO}_{2}$ substrate surface at ambient temperature, respectively, and then kept them at $70{ }^{\circ} \mathrm{C}$ in the oven for $6.0 \mathrm{~h}$ to evaporate the solvent. The absorption and fluorescence emission spectra of these three films in the dry state were shown in Supplementary Fig. 144-146. H1-functionalized polymer film $\left(\lambda_{\mathrm{em}}=508 \mathrm{~nm}\right)$ displayed blue-shifted emission when being compared with metallacycle $\mathbf{H 1}$ in solution $\left(\lambda_{\mathrm{em}}=487 \mathrm{~nm}\right)$, however, both $\mathbf{H 3}$ and $\mathbf{H 7}$-functionalized polymer films $\left(\lambda_{\mathrm{em}}=\right.$ $576 \mathrm{~nm}$ for $\mathbf{H 3}, \lambda_{\mathrm{em}}=516 \mathrm{~nm}$ for $\mathbf{H 7}$ ) exhibited an obvious blueshifted emission when compared with metallacycles $\mathbf{H 3}$ and $\mathbf{H 7}$ in solution $\left(\lambda_{\mathrm{em}}=586 \mathrm{~nm}\right.$ for $\mathbf{H 3}, \lambda_{\mathrm{em}}=555 \mathrm{~nm}$ for H7). It might be attributed to the different aggregation behaviors of metallacycles H1, H3, and H7 within the film. Notably, H1, H3, and $\mathbf{H 7}$-functionalized polymer films emitted blue fluorescence, orange fluorescence, and green fluorescence with high fluorescence quantum yield up to $29 \%, 18 \%$, and $23 \%$, respectively. Moreover, by taking advantage of the easy processing and good elasticity of polymer film, 3-D fish-shape fluorescent films exhibiting bright fluorescence with different colors were fabricated by hand (Fig. 8b-g). Moreover, fluorescent films fabricated from metallacycles and ligands exhibited different fluorescence properties (Supplementary Fig. 148). For example, 3-D fish-shape fluorescent films prepared from ligands L1 displayed green fluorescence while the fluorescent films made from metallacycle H1 emitted blue fluorescence with the stronger emission.

Inkjet printing, as a cheap, easily handled yet powerful technique for creating highly-defined patterns, has been used for conductive circuits, flexible electronics, and fingerprint recognition ${ }^{61-63}$. So far, single-walled carbon nanotubes, luminescent CdTe nanocrystals, carbon dots, and fluorescein have been patterned using this method ${ }^{64,65}$. However, to the best of 


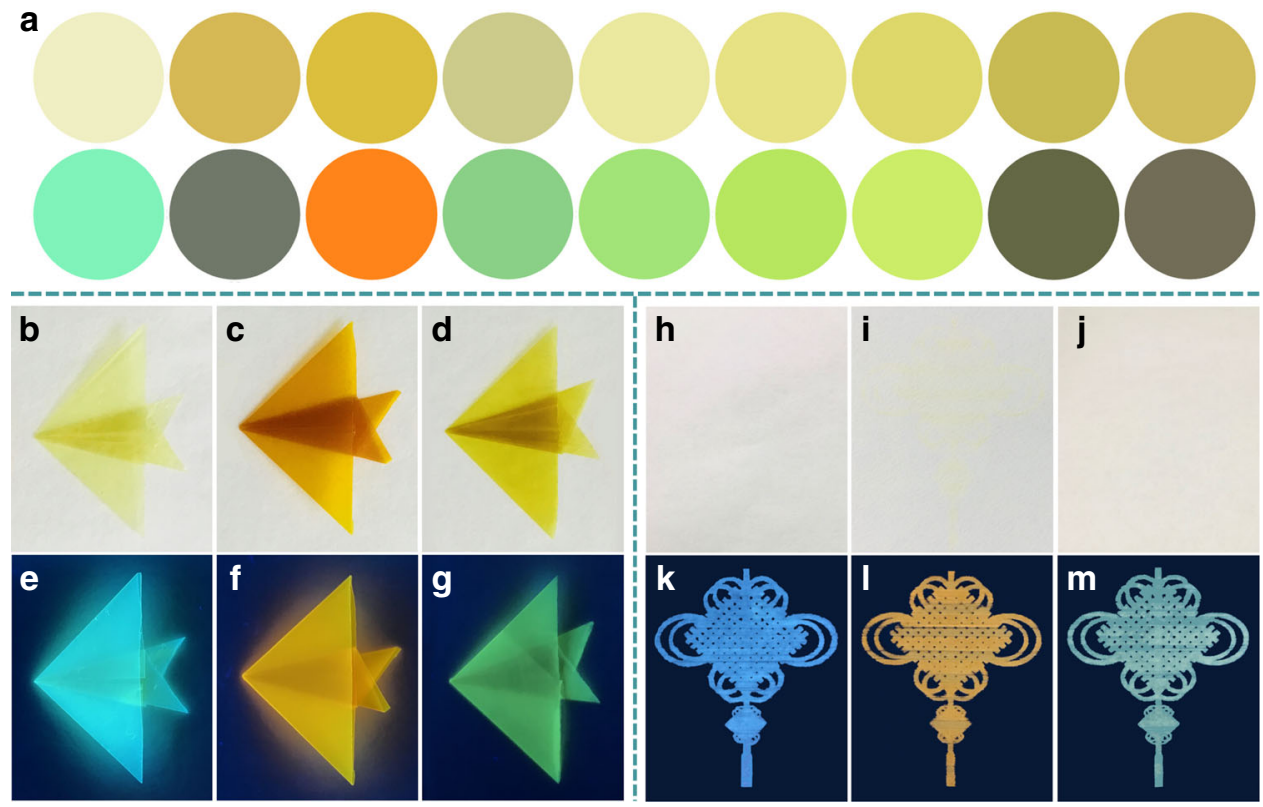

Fig. 8 Versatile fluorescent materials. a Visual (top) and fluorescence colors (bottom) of metallacycles H1-H9-coated TLC plates (Figures showed from left to right were sequentially corresponded to metallacycles H1-H9). The fluorescence colors were observed by using a hand-held UV lamp with an excitation at $365 \mathrm{~nm}$. Photographs of the flexible fluorescent films $\mathbf{b}-\mathbf{g}$ and the fluorescent patterns $\mathbf{h}-\mathbf{m}$ by inkjet printing from metallacycle $\mathbf{H} \mathbf{1}$ in visible light $\mathbf{b}$, $\mathbf{h}$ and under $365 \mathrm{~nm}$ light excitation e, $\mathbf{k}$, metallacycle $\mathbf{H 3}$ in visible light $\mathbf{c}$, i and under $365 \mathrm{~nm}$ light excitation f, I, metallacycle $\mathbf{H 7}$ in visible light $\mathbf{d}, \mathbf{j}$ and under $365 \mathrm{~nm}$ light excitation $\mathbf{g}$, $\mathbf{m}$

our knowledge, the use of SCCs for fluorescent inkjet printing has not been reported. Thus, herein, metallacycles $\mathbf{H 1}, \mathbf{H} 3$, and H7 were also selected as the representatives to explore the application as fluorescent inks for printing versatile fluorescent patterns. The acetone solution of metallacycles $\mathbf{H 1}, \mathbf{H 3}$, and $\mathbf{H 7}$ as the inkjetprinting source was printed, respectively. As shown in Fig. $8 \mathrm{~h}-\mathrm{m}$, all of the patterns printed from metallacycles $\mathbf{H 1}, \mathbf{H 3}$, and $\mathbf{H 7}$ solutions were invisible under ambient conditions, which resulted in the information of the patterns being unrecognizable by the naked eye. However, it should be noted that three patterns of the Chinese knot showing blue fluorescence, orange fluorescence, and blue-green fluorescence could be observed clearly under the excitation of hand-held UV lamp $(365 \mathrm{~nm})$, respectively. The results suggested that all kinds of patterns with multicolor fluorescence and designable shapes could be printed using different types of ink prepared from metallacycles H1-H9. Moreover, it should be noted that the patterns printed from metallacycles displayed different fluorescence colors compared to the patterns from simple model metal complexes and organic fluorescence ligands (Supplementary Fig. 147-148). This finding further illustrated that PET/ICT regulation strategy was an effective approach to construct organoplatinum metallacycles with tunable fluorescence wavelengths not only in solution but also in solid state. Therefore, the "vis-invisible" and "UV-visible" properties of metallacycles $\mathbf{H 1}, \mathbf{H 3}$, and $\mathbf{H 7}$ may allow for their potential applications in anti-counterfeiting, information hiding and storage, and optoelectronic devices.

\section{Discussion}

In summary, we presented the example on the construction of organoplatinum metallacycles with high fluorescence quantum yields and tunable fluorescence wavelengths by simply switching the PET and ICT properties of building blocks based on substituent effect. In this study, nine $120^{\circ}$ triarylamine-based dipyridyl donor ligands L1-L9 with different pendant functional groups (including $-\mathrm{CF}_{3},-\mathrm{NO}_{2},-\mathrm{CH}=\mathrm{C}(\mathrm{CN})_{2},-\mathrm{CHO},-\mathrm{H},-\mathrm{CH}_{3},-\mathrm{OCH}_{3}$,
$-\mathrm{NH}_{2}$, and $\left.-\mathrm{N}\left(\mathrm{CH}_{3}\right)_{2}\right)$ para to the tertiary amine core were designed and synthesized, from which a series of hexagonal organoplatinum metallacycles $\mathbf{H 1 - H 9}$ with different substituents were successfully prepared through coordination-driven self-assembly.

The photophysical properties investigation of metallacycles H1-H9 revealed that metallacycles $\mathbf{H 1 - H 4}$ with electronwithdrawing groups para to the tertiary amine core displayed the higher emission intensity (with the fluorescence quantum yield up to 61\%) compared with their constituent ligands L1-L4, however, the metallacycles $\mathbf{H 5 - H 9}$ containing electron-donating groups para to the tertiary amine core exhibited the lower emission intensity compared with their constituent ligands L5L9. Moreover, metallacycles H1-H9 exhibited tunable fluorescence wavelengths ranging from $480 \mathrm{~nm}$ to $590 \mathrm{~nm}$ and emitted different fluorescence colors in the same solvent. The luminescence mechanisms of these metallacycles H1-H9 were systematically investigated by 3-D EEMs, TCSPC method, TD-DFT calculation, and control experiments as well. The results indicated that the PET and ICT properties of metallacycles could be switched by the modification of substituents, which offered the selfassembled organoplatinum metallacycles with high fluorescence quantum yields and tunable fluorescence wavelengths.

By utilizing the excellent fluorescence properties of resultant metallacycles, many kinds of fluorescent materials including fluorescent metallacycle-loaded TLC plates, 3-D-fish-shape fluorescent films, and fluorescent inks for inkjet printing were successfully prepared. Notably, these metallacycle-functionalized polymer films emitted bright fluorescence with different colors and relatively high fluorescence quantum yield up to $29 \%$. Moreover, three patterns of the Chinese knot with multicolor fluorescence and designable shapes were printed using different types of ink prepared from metallacycles. The "vis-invisible" and "UV-visible" properties of these prepared fluorescent materials offer them wide potential applications in anti-counterfeiting, information hiding and storage, and optoelectronic devices. In conclusion, this study presents the example on the construction of organoplatinum metallacycles with high fluorescence quantum yields and tunable fluorescence wavelengths by 
simply switching the PET and ICT properties of building blocks based on the substituent effect.

\section{Methods}

General information. All reagents were commercially available and used without further purification. TLC analyses were performed on silica-gel plates, and flash chromatography was conducted using silica-gel column packages. All air-sensitive reactions were carried out under argon atmosphere. ${ }^{1} \mathrm{H} \mathrm{NMR},{ }^{13} \mathrm{C} \mathrm{NMR}$, and ${ }^{31} \mathrm{P}$ NMR spectra were recorded on a Bruker $300 \mathrm{MHz}$ spectrometer $\left({ }^{1} \mathrm{H}, 300 \mathrm{MHz}\right)$, a Bruker $400 \mathrm{MHz}$ spectrometer $\left({ }^{1} \mathrm{H}, 400 \mathrm{MHz},{ }^{13} \mathrm{C}, 100 \mathrm{MHz},{ }^{31} \mathrm{P}, 161.9 \mathrm{MHz}\right)$, and a Bruker $500 \mathrm{MHz}$ spectrometer $\left({ }^{1} \mathrm{H}, 500 \mathrm{MHz},{ }^{13} \mathrm{C}, 126 \mathrm{MHz},{ }^{31} \mathrm{P}, 202 \mathrm{MHz}\right)$ at $298 \mathrm{~K}$. The ${ }^{1} \mathrm{H}$ and ${ }^{13} \mathrm{C}$ NMR chemical shifts are reported relative to residual solvent signals, and ${ }^{31} \mathrm{P}$ NMR resonances are referenced to internal standard sample of $85 \% \mathrm{H}_{3} \mathrm{PO}_{4}(\delta 0.0)$.

General procedure for preparation of H1-H9. The dipyridyl donor ligand L1-L9 $(6.0 \mu \mathrm{mol})$ and the organoplatinum $120^{\circ}$ acceptor $8(6.0 \mu \mathrm{mol})$ were weighed accurately into a glass vial. To the vial was added $2.0 \mathrm{~mL}$ acetone and the reaction solution was then stirred at $40^{\circ} \mathrm{C}$ for $4 \mathrm{~h}$ to yield a homogeneous solution (for L8, $2.0 \mathrm{~mL}$ methanol was added into the vial and the reaction solution was stirred at $60{ }^{\circ} \mathrm{C}$ for $4 \mathrm{~h}$ ). Solid product H1-H9 was obtained by removing the solvent under vacuum.

\section{UV-Vis absorption, fluorescence emission and excitation spectra. UV-vis} spectra were recorded in a quartz cell (light path $10 \mathrm{~mm}$ ) on a Cary 50Bio UV -visible spectrophotometer. Steady-state fluorescence spectra and excitation spectra were recorded in a conventional quartz cell (light path $2 \mathrm{~mm}$ ) on a Cary Eclipse fluorescence spectrophotometer.

3-D EEM and lifetime experiment. Three-dimensional excitation-emission matrix spectra were recorded in a quartz cell (light path $2 \mathrm{~mm}$ ) on a HORIBA FluoroMax-4 fluorescence spectrophotometer.

Lifetime were measured on a home-made system based on the TCSPC technique ${ }^{66}$

Fluorescence quantum yields. Fluorescence quantum yields were measured in absolutely in solution using a commercial fluorometer with integrating sphere (RF6000, shimadzu).

Fluorescence lifetimes. The fluorescence lifetimes were measured based on TCSPC technique. The squared deviations obtained by the least-squares analysis was utilized to estimate the fitting of TCSPC data and judge the multiexponential emission decay processes.

DFT and TD-DFT calculations. All calculations were performed using the Gaussian09 (G09) program package. The DFT method was employed using the B3LYP hybrid functional with LANL2DZ effective core potentials $(\mathrm{Pt})$ and $6-31 \mathrm{G}^{*}$ $(\mathrm{C}, \mathrm{H}, \mathrm{N}, \mathrm{F}$ and $\mathrm{O}$ ) basis set. Orbitals were visualized using GaussView 5.0 with an isovalue of 0.02 . To minimize computational cost, we simplified the compound and replaced it with $1 / 3$ of the compound.

Geometry optimizations. The compound $\mathbf{H 1 - H 9}$ were optimized by the PM6 semiempirical molecular orbital method. And the ligand L1-L9 were optimized by the DFT method.

\section{Data availability}

The data that support the findings of this study are available from the authors on reasonable request, see author contributions for specific data sets. The X-ray crystallographic coordinates for structures reported in this study have been deposited at the Cambridge Crystallographic Data Centre (CCDC), under deposition numbers $1873682,1873683,1873684$, and 1873685 . These data can be obtained free of charge from The Cambridge Crystallographic Data Centre via www.ccdc.cam.ac.uk/ data_request/cif.

Received: 27 December 2018 Accepted: 27 August 2019 Published online: 19 September 2019

\section{References}

1. Cook, T. R. \& Stang, P. J. Recent developments in the preparation and chemistry of metallacycles and metallacages via coordination. Chem. Rev. 115, 7001-7045 (2015).
2. Fujita, M., Tominaga, M., Hori, A. \& Therrien, B. Coordination assemblies from a Pd(II)-cornered square complex. Acc. Chem. Res. 38, 369-378 (2005).

3. Brown, C. J., Toste, F. D., Bergman, R. G. \& Raymond, K. N. Supramolecular catalysis in metal-ligand cluster hosts. Chem. Rev. 115, 3012-3035 (2015).

4. Chakraborty, S. \& Newkome, G. R. Terpyridine-based metallosupramolecular constructs: tailored monomers to precise 2D-motifs and 3D-metallocages. Chem. Soc. Rev. 47, 3991-4016 (2018).

5. Yoshizawa, M. \& Klosterman, J. K. Molecular architectures of multianthracene assemblies. Chem. Soc. Rev. 43, 1885-1898 (2014).

6. Han, M., Engelhard, D. M. \& Clever, G. H. Self-assembled coordination cages based on banana-shaped ligands. Chem. Soc. Rev. 43, 1848-1860 (2014).

7. Zarra, S., Wood, D. M., Roberts, D. A. \& Nitschke, J. R. Molecular containers in complex chemical systems. Chem. Soc. Rev. 44, 419-432 (2015).

8. Lu, Y., Zhang, H.-N. \& Jin, G.-X. Molecular borromean rings based on halfsandwich organometallic rectangles. Acc. Chem. Res. 51, 2148-2158 (2018).

9. Lescop, C. Coordination-driven syntheses of compact supramolecular metallacycles toward extended metallo-organic stacked supramolecular assemblies. Acc. Chem. Res. 50, 885-894 (2017).

10. Chen, L.-J., Yang, H.-B. \& Shionoya, M. Chiral metallosupramolecular architectures. Chem. Soc. Rev. 46, 2555-2576 (2017).

11. Howlader, P., Mondal, B., Purba, P. C., Zangrando, E. \& Mukherjee, P. S. Selfassembled Pd(II) barrels as containers for transient merocyanine form and reverse thermochromism of spiropyran. J. Am. Chem. Soc. 140, 7952-7960 (2018).

12. Omoto, K., Tashiro, S., Kuritani, M. \& Shionoya, M. Multipoint recognition of ditopic aromatic guest molecules via $\mathrm{Ag}-\pi$ interactions within a dimetal macrocycle. J. Am. Chem. Soc. 136, 17946-17949 (2014).

13. Yazaki, K. et al. Polyaromatic molecular peanuts. Nat. Commun. 8, 15914-15921 (2017)

14. Wang, J., He, C., Wu, P., Wang, J. \& Duan, C. An amide-containing metal-organic tetrahedron responding to a spin-trapping reaction in a fluorescent enhancement manner for biological imaging of NO in living cells. J. Am. Chem. Soc. 133, 12402-12405 (2011).

15. Chen, L.-J. et al. Hierarchical self-assembly of discrete organoplatinum(II) metallacycles with polysaccharide via electrostatic interactions and their application for heparin detection. J. Am. Chem. Soc. 137, 11725-11735 (2015).

16. Chowdhury, A., Howlader, P. \& Mukherjee, P. S. Aggregation-induced emission of platinum(II) metallacycles and their ability to detect nitroaromatics. Chem. Eur. J. 22, 7468-7478 (2016).

17. Yan, K. \& Fujita, M. A speedy marriage in supramolecular catalysis. Science 350, 1165-1166 (2015).

18. Oldacre, A. N., Friedman, A. E. \& Cook, T. R. A self-assembled cofacial cobalt porphyrin prism for oxygen reduction catalysis. J. Am. Chem. Soc. 139, 1424-1427 (2017)

19. Howlader, P., Das, P., Zangrando, E. \& Mukherjee, P. S. Urea-functionalized self-assembled molecular prism for heterogeneous catalysis in water. J. Am. Chem. Soc. 138, 1668-1676 (2016).

20. Wang, Q.-Q. et al. Self-assembled nanospheres with multiple endohedral binding sites pre-organize catalysts and substrates for highly efficient reactions. Nat. Chem. 8, 225-230 (2016).

21. Kaphan, D. M., Toste, F. D., Bergman, R. G. \& Raymond, K. N. Enabling new modes of reactivity via constrictive binding in a supramolecular-assemblycatalyzed aza-prins cyclization. J. Am. Chem. Soc. 137, 9202-9205 (2015).

22. Yan, X. et al. Responsive supramolecular polymer metallogel constructed by orthogonal coordination-driven self-assembly and host/guest interactions. J. Am. Chem. Soc. 136, 4460-4463 (2014).

23. Zheng, W. et al. Construction of smart supramolecular polymeric hydrogels cross-linked by discrete organoplatinum(II) metallacycles via post-assembly polymerization. J. Am. Chem. Soc. 138, 4927-4937 (2016).

24. Cook, T. R., Vajpayee, V., Lee, M. H., Stang, P. J. \& Chi, K.-W. Biomedical and biochemical applications of self-assembled metallacycles and metallacages. Acc. Chem. Res. 46, 2464-2474 (2013).

25. Yue, Z. et al. Coordination-driven self-assembly of a $\mathrm{Pt}(\mathrm{IV})$ prodrugconjugated supramolecular hexagon. Chem. Commun. 54, 731-734 (2018).

26. Zhou, Z. et al. Heterometallic Ru-Pt metallacycle for two-photon photodynamic therapy. Proc. Natl. Acad. Sci. USA 115, 5664-5669 (2018).

27. Yan, X., Cook, T. R., Wang, P., Huang, F. \& Stang, P. J. Highly emissive platinum(II) metallacages. Nat. Chem. 7, 342-348 (2015).

28. Harano, K., Hiraoka, S. \& Shionoya, M. 3 nm-scale molecular switching between fluorescent coordination capsule and nonfluorescent cage. J. Am. Chem. Soc. 129, 5300-5301 (2007).

29. Kaloudi-Chantzea, A. et al. Coordination-driven self assembly of a brilliantly fluorescent rhomboid cavitand composed of bodipy-dye subunits. J. Am. Chem. Soc. 132, 16327-16329 (2010).

30. Liu, C.-L. et al. Intraligand charge transfer sensitization on self-assembled europium tetrahedral cage leads to dual-selective luminescent sensing toward anion and cation. J. Am. Chem. Soc. 139, 12474-12479 (2017). 
31. Yin, G.-Q. et al. Self-assembly of emissive supramolecular rosettes with increasing complexity using multitopic terpyridine ligands. Nat. Commun. 9, 567-576 (2018).

32. Zhang, R.-L. et al. Direct observation of a triplet-state absorption-emission conversion in a fullerene-functionalized Pt(II) metallacycle. J. Phys. Chem. C. 121, 14975-14980 (2017).

33. Zhang, R.-L., Yang, Y., Yang, S.-Qiu \& Han, K.-L. Unveiling excited state energy transfer and charge transfer in a host/guest coordination cage. Phys. Chem. Chem. Phys. 20, 2205-2210 (2018).

34. Ono, K. et al. ON/OFF red emission from azaporphine in a coordination cage in water. J. Am. Chem. Soc. 131, 12526-12527 (2009).

35. Li, Z., Kishi, N., Yoza, K., Akita, M. \& Yoshizawa, M. Isostructural $M_{2} L_{4}$ molecular capsules with anthracene shells: synthesis, crystal structures, and fluorescent properties. Chem. Eur. J. 18, 8358-8365 (2012).

36. Neelakandan, P. P., Jiménez, A. \& Nitschke, J. R. Fluorophore incorporation allows nanomolar guest sensing and white-light emission in $\mathrm{M}_{4} \mathrm{~L}_{6}$ cage complexes. Chem. Sci. 5, 908-915 (2014)

37. Tsutsui, T., Kusaba, S., Yamashina, M., Akita, M. \& Yoshizawa, M. Open versus closed polyaromatic nanocavity: enhanced host abilities toward large dyes and pigments. Chem. Eur. J. 25, 4320-4324 (2019).

38. Huang, C.-B. et al. Real-time monitoring the dynamics of coordination-driven self-assembly by fluorescence-resonance energy transfer. J. Am. Chem. Soc. 139, 9459-9462 (2017).

39. Bar, A. K., Chakrabarty, R., Mostafa, G. \& Mukherjee, P. S. Self-assembly of a nanoscopic $\mathrm{Pt}_{12} \mathrm{Fe}_{12}$ heterometallic open molecular box containing six porphyrin walls. Angew. Chem. Int. Ed. 47, 8455-8459 (2008).

40. Shi, Y., Sánchez-Molina, I., Cao, C., Cook, T. R. \& Stang, P. J. Synthesis and photophysical studies of self-assembled multicomponent supramolecular coordination prisms bearing porphyrin faces. Proc. Natl. Acad. Sci. USA 111, 9390-9395 (2014).

41. Yamashina, M. et al. Preparation of highly fluorescent host-guest complexes with tunable color upon encapsulation. J. Am. Chem. Soc. 137, 9266-9269 (2015).

42. Roy, B., Ghosh, A. K., Srivastava, S., D’Silva, P. \& Mukherjee, P. S. A Pd tetrafacial molecular barrel as carrier for water insoluble fluorophore. J. Am. Chem. Soc. 137, 11916-11919 (2015).

43. Chen, J.-S., Zhao, G.-J., Cook, T. R., Han, K.-L. \& Stang, P. J. Photophysical properties of self-assembled multinuclear platinum metallacycles with different conformational geometries. J. Am. Chem. Soc. 135, 6694-6702 (2013).

44. Pollock, J. B., Cook, T. R. \& Stang, P. J. Photophysical and computational investigations of bis (phosphine) organoplatinum(II) metallacycles. J. Am. Chem. Soc. 134, 10607-10620 (2012).

45. Zhang, M. et al. Metallacycle-cored supramolecular assemblies with tunable fluorescence including white-light emission. Proc. Natl. Acad. Sci. USA 114, 3044-3049 (2017).

46. Wen, Y. et al. Introduction of red-green-blue fluorescent dyes into a metalorganic framework for tunable white light emission. Adv. Mater. 29, 1700778 (2017).

47. Kim, J.-H., Jung, Y., Lee, D. \& Jang, W.-D. Thermoresponsive polymer and fluorescent dye hybrids for tunable multicolor emission. Adv. Mater. 28, 3499-3503 (2016).

48. Mao, Z. et al. Linearly tunable emission colors obtained from a fluorescentphosphorescent dual-emission compound by mechanical stimuli. Angew. Chem. Int. Ed. 54, 6270-6273 (2015).

49. de Silva, A. P. Luminescent photoinduced electron transfer (PET) molecules for sensing and logic operations. J. Phys. Chem. Lett. 2, 2865-2871 (2011)

50. Li, Y., Liu, T., Liu, H., Tian, M.-Z. \& Li, Y. Self-assembly of intramolecular charge-transfer compounds into functional molecular systems. Acc. Chem. Res. 47, 1186-1198 (2014).

51. Cheng, T. et al. A highly sensitive and selective OFF-ON fluorescent sensor for cadmium in aqueous solution and living cell. J. Am. Chem. Soc. 130, 16160-16161 (2008).

52. Sun, X., James, T. D. \& Anslyn, E. V. Arresting "Loose Bolt" internal conversion from $-\mathrm{B}(\mathrm{OH})_{2}$ groups is the mechanism for emission turn-on in ortho-aminomethylphenylboronic acid-based saccharide sensors. J. Am. Chem. Soc. 140, 2348-2354 (2018).

53. Zhang, H., Hu, J. \& Qu, D.-H. Dual-mode control of PET process in a ferrocene-functionalized [2] rotaxane with high-contrast fluorescence output. Org. Lett. 14, 2334-2337 (2012).

54. Denis, M., Pancholi, J., Jobe, K., Watkinson, M. \& Goldup, S. M. Chelating rotaxane ligands as fluorescent sensors for metal ions. Angew. Chem. Int. Ed. 57, 5310-5314 (2018).

55. Crespo-Hernández, C. E., Burdzinski, G. \& Arce, R. Environmental photochemistry of nitro-PAHs: direct observation of ultrafast intersystem crossing in 1-nitropyrene. J. Phys. Chem. A 112, 6313-6319 (2008).
56. Zhao, X. et al. An aggregation-induced emission-based "Turn-On" fluorescen probe for facile detection of gaseous formaldehyde. ACS Sens. 3, 2112-2117 (2018).

57. Cui, L. et al. Unique Tri-output optical probe for specific and ultrasensitive detection of hydrazine. Anal. Chem. 86, 4611-4617 (2014).

58. Nie, H. et al. Tetraphenylfuran: aggregation-induced emission or aggregationcaused quenching. Mater. Chem. Front. 1, 1125-1129 (2017).

59. $\mathrm{Ma}, \mathrm{H}$. et al. A dendrimer-based electropolymerized microporous film: multifunctional, reversible, and highly sensitive fluorescent probe. Adv. Funct. Mater. 26, 2025-2031 (2016).

60. Wang, Z. et al. A novel mechanochromic and photochromic polymer film: when rhodamine joins polyurethane. Adv. Mater. 27, 6469-6474 (2015).

61. Yamada, K., Henares, T. G., Suzuki, K. \& Citterio, D. Paper-based inkjet-printed microfluidic analytical devices. Angew. Chem. Int. Ed. 54, 5294-5310 (2015).

62. Qin, Y., Alam, A. U., Howlader, M. M. R., Hu, N.-X. \& Deen, M. J. Inkjet printing of a highly loaded palladium ink for integrated, low-cost $\mathrm{pH}$ sensors. Adv. Funct. Mater. 26, 4923-4933 (2016).

63. Gao, Z., Han, Y. \& Wang, F. Cooperative supramolecular polymers with anthracene-endoperoxide photo-switching for fluorescent anti-counterfeiting Nat. Commun. 9, 3977-3985 (2018).

64. Bucella, S. G. et al. Inkjet printed single-walled carbon nanotube based ambipolar and unipolar transistors for high-performance complementary logic circuits. Adv. Electron. Mater. 2, 1600094 (2016).

65. Tekin, E. et al. Inkjet printing of luminescent CdTe nanocrystal-polymer composites. Adv. Funct. Mater. 17, 23-28 (2006).

66. Liu, M. et al. Instrument response standard in time-resolved fluorescence spectroscopy at visible wavelength: quenched fluorescein sodium. Appl. Spectrosc. 68, 577-583 (2014).

\section{Acknowledgements}

This work was financially supported by the NSFC/China (Nos. 21625202, 21672070, 21922506, and 21871092), and Shanghai Pujiang Program (No. 18PJD015). We thankfully acknowledge Professor Feng Wang of University of Science and Technology of China for the discussion of fluorescent inkjet printing, Professor Haitao Sun (ECNU) and Mr. Zhubin Hu (ECNU) for the TD-DFT calculations, and Mr. Changxing Zhao of East China University of Science and Technology for the discussion of phosphorescence experiment.

\section{Author contributions}

H.-B.Y., L.X. and J.-L.Z. conceived the project, analyzed the date, and wrote the manuscript. J.-L.Z. performed the most of experiments. J.-L.Z. and Y.-Y.R. completed the synthesis. Y.-Y.R., Y.Z., X.L., G.-Q. Y., B.S., X.C., Z.C., X.-L.Z., H.T., J.C. and X.-P.L. helped in experiments and data analyses. All authors discussed the results and commented on the manuscript.

\section{Additional information}

Supplementary Information accompanies this paper at https://doi.org/10.1038/s41467 019-12204-7.

Competing interests: The authors declare no competing interests.

Reprints and permission information is available online at http://npg.nature.com/ reprintsandpermissions/

Peer review information Nature Communications thanks the anonymous reviewer(s) for their contribution to the peer review of this work. Peer reviewer reports are available.

Publisher's note Springer Nature remains neutral with regard to jurisdictional claims in published maps and institutional affiliations.

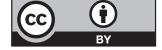

Open Access This article is licensed under a Creative Commons Attribution 4.0 International License, which permits use, sharing, adaptation, distribution and reproduction in any medium or format, as long as you give appropriate credit to the original author(s) and the source, provide a link to the Creative Commons license, and indicate if changes were made. The images or other third party material in this article are included in the article's Creative Commons license, unless indicated otherwise in a credit line to the material. If material is not included in the article's Creative Commons license and your intended use is not permitted by statutory regulation or exceeds the permitted use, you will need to obtain permission directly from the copyright holder. To view a copy of this license, visit http://creativecommons.org/ licenses/by/4.0/

(C) The Author(s) 2019 